\title{
Silicon Light-Emitting Device with Application in on-Chip Micro-opto-electro-mechanical and Chemical-opto-electro Micro Systems
}

\author{
Kaikai Xu, Lukas W. Snyman, Jean-Luc Polleux, Hongda Chen, and Guannpyng Li
}

\begin{abstract}
The advances in silicon photonics related device development have been evolved into standard complementary metal-oxide-semiconductor (CMOS) technology in recent years. The emission of visible light $(400-900 \mathrm{~nm})$ by a monolithically integrated silicon $p-n$ junction under reverse bias presents the silicon light-emitting device (Si-LED). As an integrated optical source, it is then developed for coupling light into the optic waveguide. Through the Monte Carlo and Rsoft BeamPROP simulations, the vertical emission, focusing, refraction, splitting and wave-guiding are also optimized using the same CMOS technology. Since the $\mathrm{Si}$-LED, the $\mathrm{SiO}_{2}$-waveguide, and the Si-photodetector can be monolithically integrated on the same bulk-Si substrate, a concise micro-opto-electro-mechanical systems (MOEMS) could be realized in the modern CMOS structural \& integrated circuitry standard platform.
\end{abstract}

Index Terms-Silicon LED, micro-opto-electro-mechanical systems, waveguide, CMOS technology, monolithic integration.

\section{INTRODUCTION}

The exponential growth of global data volumes and associated data centers is outpacing the industry's ability to efficiently produce more powerful data processing integrated circuits in accordance with Moore's Law. The modern trend of deploying more parallel computers with higher capacity data storage solves the problem of lagging microprocessor speeds, but the resultant significant proliferation of separate data computing equipment in turn leads to a growing need for faster and more efficient communication between computers and data storage. Hence, the material of silicon based

Manuscript received April 12, 2015; revised May 20, 2015. This work was supported by the Fundamental Research Funds for the Central Universities under Cooperative Agreement ZYGX2015KYQD009, Ministry of Education, China, the South Africa National Research Foundation Grant FA200604110043, the Calit2 Fellowship, and a Key International Collaboration Grant KSC 69798 in South Africa.

K. Xu is with the the State Key Laboratory of Electronic Thin Films and Integrated Devices, University of Electronic Science and Technology of China, Chengdu, Sichuan 610054 China (e-mail: kaikaix@uestc.edu.cn).

L. Snyman is with the Department of Electrical and Mining Engineering, University of South Africa, Pretoria 0001 South Africa (e-mail: snymalw@unisa.ac.za).

J. Polleux is with the University Paris-Est, ESYCOM, ESIEE Paris, Le Cnam, UPEM, Cite Descartes, Noisy-Le-Grand-Cedex 93162 France (e-mail: jean-luc.polleux@esiee.fr).

H. Chen is with the State Key Laboratory on Integrated Optoelectronics, Institute of Semiconductors, Chinese Academy of Sciences, Beijing 100083 China (e-mail: hdchen@semi.ac.cn).

$\mathrm{G}$. $\mathrm{Li}$ is with the California Institute for Telecommunications and Information Technology, Irvine 92697 California, USA. (e-mail: gpli@uci.edu). optoelectronics is considered to enable future computing systems with optical input-outputs co-packaged with CMOS chips to circumvent the limitation of electrical interface.

Despite of the indirect bandgap in $\mathrm{Si}$, a series of viable $\mathrm{Si}$ light-emitting technologies have recently become available that enable the integration of light sources directly into $\mathrm{Si}$ CMOS technology [1]. The latest attempts for realizing optoelectronic systems in CMOS technology have until now mainly been focused utilizing wavelengths at $1550 \mathrm{~nm}$, mainly because of the ease of design and fabrication of waveguides in this regime [2]. However, no effective optical sources and $\mathrm{Si}$ detectors are available at this wavelength.

Being different from the silicon photonics work that is done at wavelengths around $1550 \mathrm{~nm}$, it is observed that visible light with a typical spectral distribution curve in the wavelength range of 365 to $689 \mathrm{~nm}$ is emitted by silicon p-n junctions both in avalanche breakdown and in breakdown by internal field emission [3]. The light emission implies that $\mathrm{Si}$ diode under avalanche breakdown condition is a potential light transmitter which can monolithically integrate with silicon electronic circuits using the standard S-CMOS process [4], since the light source is a silicon device. On the other hand, a need for developing shorter wavelength optical sources $(\sim 450 \mathrm{~nm})$ in order to act as an optical clock pulses in next generation CMOS circuitry has been expressed [5].

If optical source, detector, waveguides, and sensors could be realized on the same CMOS chip at the visible wavelength range, various on-chip-based micro-photonic systems can be realized. Achieving these goals can lead to diverse low cost "all-silicon" opto-electronic systems, which will be the "smarter" and more "intelligent" CMOS chips of the future. These systems could lead to new products for especially the medical and bio world. Such a new field could be appropriately named "Si CMOS photonic micro-systems." These systems also do not require ultrahigh frequency bandwidths and the emission powers of these Si light-emitting diodes (LEDs) may be sufficient to sustain the operation of such systems. This can lead to many new products and open up new markets.

This paper reviews the Si-LEDs, which are fabricated in standard CMOS process with no change to the CMOS design and processing procedures, we have jointly realized in the past two decades. By coupling the device to a standard mode optical fiber, first interation optical communication between one CMOS device and a second is successfully constructed. A new technical approach is proposed to resolve the issue of optical properties with native interband electro-optical emission in Si. Finally, the potential applications of Si-LEDs 
into micro-photonic systems and MOEMS are furthermore highlighted as a conclusion.

\section{FIELD-EFFECT ELECTROLUMINESCENCE IN SILICON}

The profile about the composition and structure of a gate-controlled diode based LED structure is shown in Fig. 1. It is a poly-Si gate p-channel MOSFET, with gate oxide thickness of $4000 \AA$. The width of the device is $\sim 175.5 \mu \mathrm{m}$ and the channel length is $\sim 6 \mu \mathrm{m}$. The poly-Si gate thickness is 400 ̊ [6].

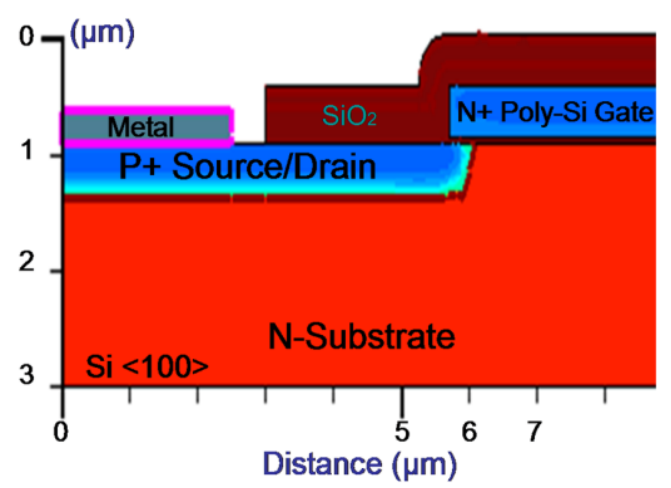

Fig. 1. Cross-section view of the a gate-controlled diode structure (i.e., one half of the Si-PMOSFET device)

In contrast, Fig. 2 shows details about the composition and structure of the planar $\mathrm{Si} n+p$ LED structure. The structure consists of a heavily doped The structure consisted of a heavily doped $n+, 10^{19} \mathrm{~cm}^{-3}$ doped layer of $0.3 \mu \mathrm{m}$ thickness defined and realized in a $0.75 \sim 1.25 \Omega \bullet \mathrm{cm}$ p-type substrate by means of appropriate ion beam implantation, masking and dopant activation procedures. Appropriate $n$-well guard-ring structures were placed on the periphery of the $n+$ region in order to ensure a uniform and planar breakdown at the planar $n+p$ interface [7].

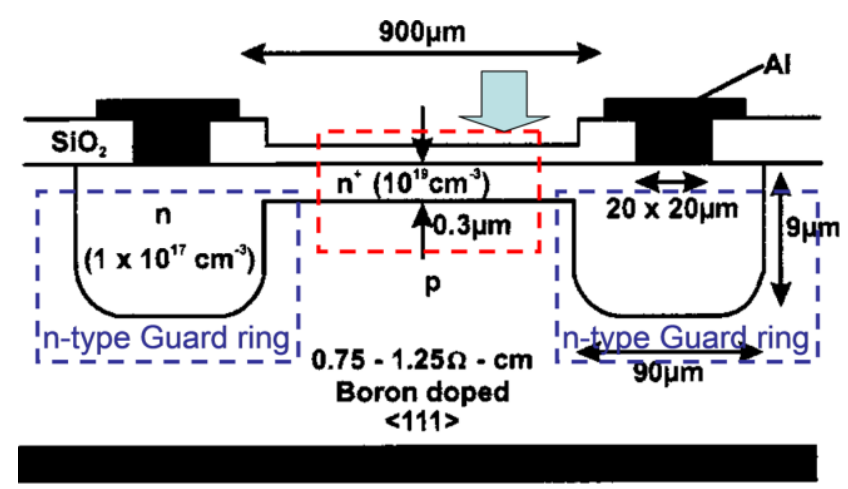

Fig. 2. Structural details of a Si $n+p$ abrupt diode (after ref. [7]).

Further, Fig. 3 presents a modified structure. When a reverse bias voltage is applied between the inner $p+$ centroid and the $n+$ ring arrangement, a lateral and concentric electrical field is created between the $p+$ centroid and the $n+$ rings, which results in the creation of extended depletion layer at each $n+p$ interface facing the $p+$ centroid. Due to the high doping concentration at the surface of the intersecting $\mathrm{Si}_{-} \mathrm{SiO}_{2}$ interface, the light is emitted vertically from the device through the thin residual $\mathrm{SiO}_{2}$ layer present on the $\mathrm{Si}$ surface [8].

\section{DESIGNING LATERAL WAVEGUIDES FOR CMOS STRUCTURES}

The current CMOS technology can create a thin oxidation layer that is used as an isolation layer in the trench technology. If the layer is enhanced and followed by a layer of high refractive index material, such as Si nitride, interesting lateral optical conductors and waveguides can be constructed at the $\mathrm{Si}$-over layer interface. Optical sources positioned at the trenches' edges enable optimum coupling of optical radiation into the trench waveguide. In a similar way waveguides can be fabricated in the outer CMOS layers.

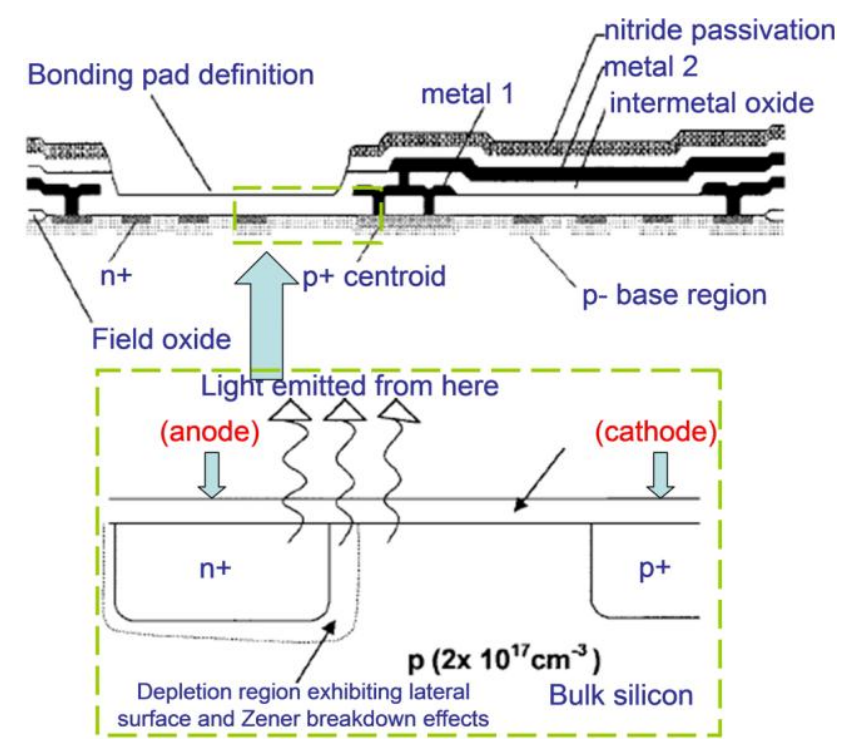

Fig. 3. Cross-section view of a Si-LED with diameter of $60 \mu \mathrm{m}$. Further design details present a schematic diagram showing the origin and the location of the light emission process at each $n+$ ring structure (after ref. [8])

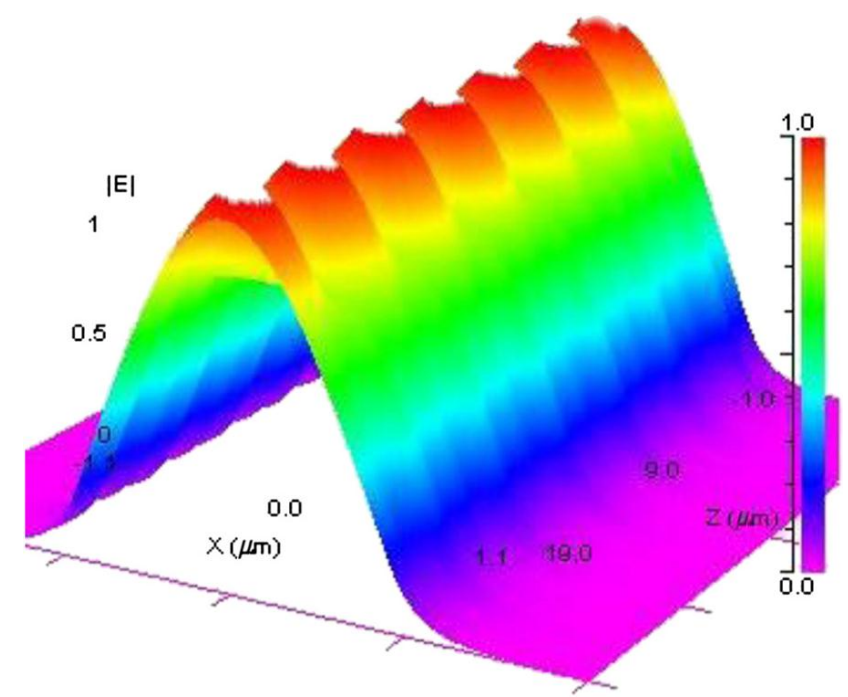

Fig. 4. Advanced optical simulation of the electrical field propagation in a 0.5 - $\mu$ m-wide $\mathrm{Si}$ nitride layer embedded in $\mathrm{SiO}_{2}$ in CMOS integrated circuitry using finite element analysis and solutions of Helmholtz's equation. Multi-mode optical propagation at $750 \mathrm{~nm}$ is demonstrated over $20 \mu \mathrm{m}$ distance with a loss smaller than $1 \mathrm{~dB} \cdot \mathrm{cm}^{-1}$.

Advanced optical simulation software (RSOFT Beam PROP) is implemented to design and simulate specific CMOS based waveguides operating at $750 \mathrm{~nm}$ based on CMOS materials and parameters. This software uses an advanced finite analysis method where the volume is divided up into a 
large number of matrix elements. Helmholtz's equation predicts the optical parameters along the matrix based on the initially defined optical fields [9].

It is preliminarily shows that a multimode, as well as single mode wave-guiding can be achieved. Fig. 4 shows a three dimensional (3-D) view of the electrical field along the waveguide of a $0.6 \mu \mathrm{m}$ wide Si nitride waveguide.

Multimode propagation is demonstrated with almost zero loss up to a distance of $20 \mu \mathrm{m}$. Multimode propagation in CMOS micro systems has the advantage of having a large acceptance angle for coupling optical radiation from a Si LED into and out of a waveguide. Our calculations show that a coupling efficiency of 0.38 can be achieved for a flat Si-emitting surface positioned at the edge of the $\mathrm{Si}$ nitride core of the waveguide. This is mainly due to the better refractive index matching between $\mathrm{Si}(3.76$ at $750 \mathrm{~nm}$ wavelength) to Si nitride (2.00 at $750 \mathrm{~nm})$.
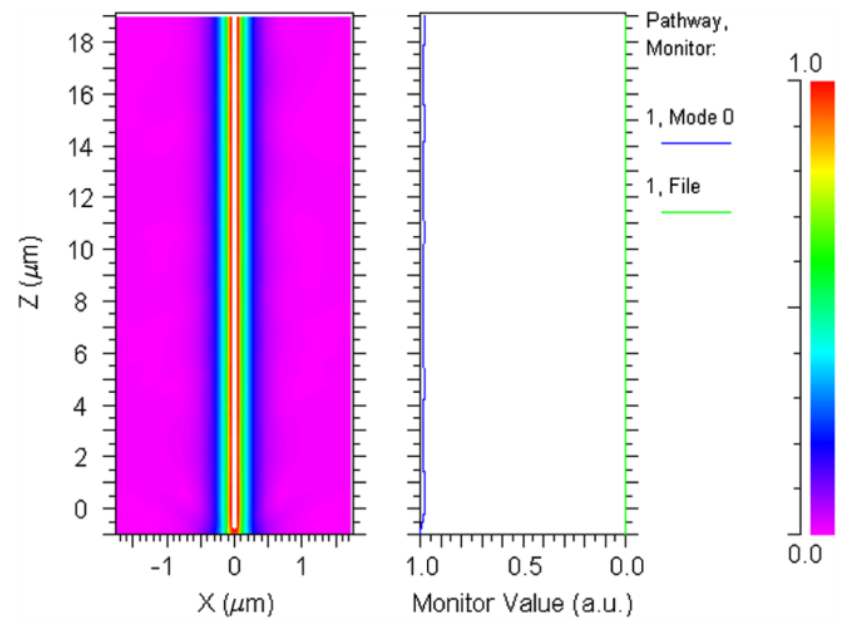

Fig. 5. Simulation of the optical field propagation in a Si nitride layer in CMOS integrated circuitry using finite element analysis and solutions of Helmholtz's equation. Single-mode optical propagation is demonstrated at $750 \mathrm{~nm}$ over a distance of 20 micron for a $0.2-\mu \mathrm{m}$ wide, Si-oxide-embedded and $\mathrm{Si}$ nitride waveguide.

Fig. 5 shows the predicted optical simulation for a $0.3-\mu \mathrm{m}$ diameter Si oxi-nitride waveguide embedded in Si oxide. The two-dimensional plot of the electrical field propagation along the waveguide shows clearly single-mode propagation. The calculated loss curve in the adjacent figure shows almost zero loss over a distance of up to $20 \mu \mathrm{m}$. The coupling efficiency into such a waveguide is, however, much reduced to values of about 0.05 . Multimode to single-mode converter structures can then be used to increase the overall coupling efficiency into the waveguide.

\section{APPliCATIONS OF Si DEVICES IN CMOS-BASED MICROPHOTONIC SYSTEMS}

A hypothetical micro-photonic system is demonstrating in Fig. 6 consists of an Si-LED, a Si detector together with waveguides integrated monolithically in a CMOS structure. Wide area Si-LEDs are used in order to increase the total optical emission power into the waveguide systems [10].

Appropriate filtering by means of ring resonators, and enhanced phase contrast detection can be obtained by utilizing unbalanced Mach-Zehnder interferometers. Near the layout end, an opening is integrated in the CMOS over layers by post processed RF etching to enable gas or liquids to interact with the evanescent field of a waveguide section and introduces intensity and/or phase contrast changes.

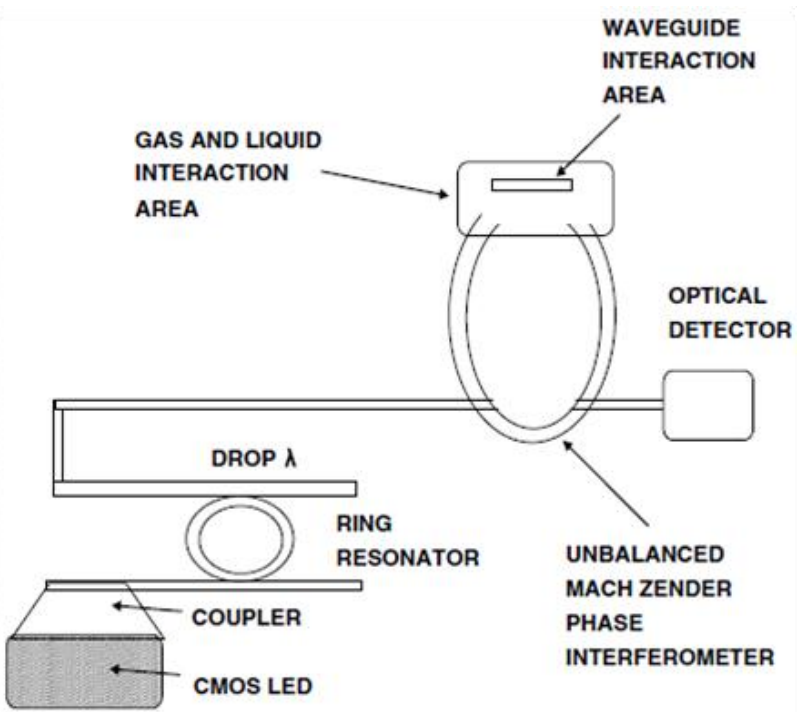

Fig. 6. Schematic diagram of a CMOS-based micro-photonic system that can be realized using an on-chip Si Av LED, a series of waveguides, ring resonators, and an unbalanced Mach-Zehnder interferometer. A section of the waveguide is exposed to the environment and can detect phase and intensity contrast due to absorption of molecules and gases in the evanescent field of the waveguide (after ref. [10]).

Hence, a complete micro-photonic sensor system can be integrated into standard CMOS circuitry. The added intelligence component, the lowering in cost and the increase in reliability of such systems can be significant. The source \& detector and waveguide arrays can be arranged to find some performances in the emerging field like bio- and nanotechnology.

\section{ANALYSIS OF ELECTROLUMINESCENCE IN Si MATERIAL}

Silicon is an indirect bandgap material, but light emission could be observed from reverse-biased pn junctions. Since electron-hole pair is produced during avalanche breakdown, some radiative recombination can occur. Both the electrons and holes can be heated by the electric field. The radiatvie transition between hot carriers emit photons larger than the bandgap. Hence, the luminescence during avalanche breakdown is characterized by a broad emission spectrum. Since the energy for impact ionization by hot carrier is about $1.5 E_{g}$, the emission spectrum extends to $\sim 3 E_{g}$ (where the energy gap $E_{g}$ is of $\sim 1.12 \mathrm{eV}$ for silicon). This represents transitions from the hottest electron energy to the hottest hole energies. In this section, several attempts are made to understand the origin of light from avalanching silicon $p-n$ junction, with physical model for the light emission.

Fig. 7 shows that, no matter what the detailed structure of the silicon pn junction is, it always emit light in a broad spectrum from 450 to $800 \mathrm{~nm}$ with characteristic peaks at 500 and $650 \mathrm{~nm}$ [4].

Based on the classical electromagnetic theory, the kinetic energy of electron is released in the form of photons if an electron collides with a singly charged Coulombic center. The 
electromagnetic field is quantized as a photonic system.

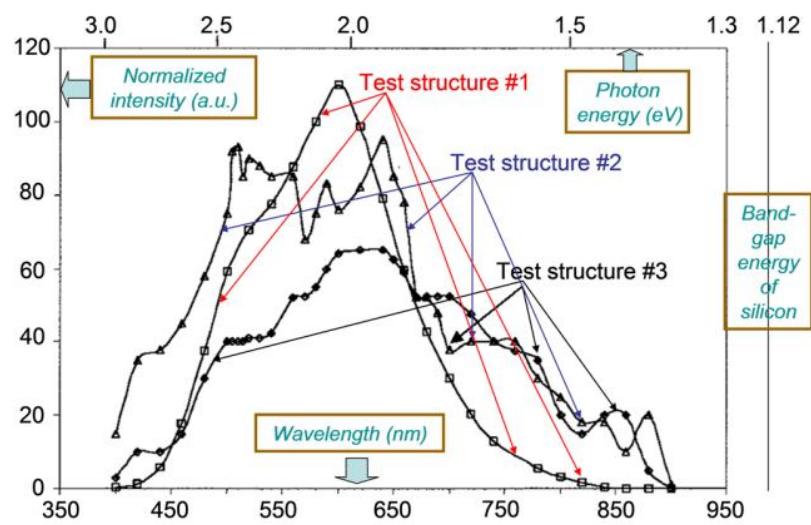

Fig. 7. The spectra of the emitted light from the several types of silicon pn junction measured at the avalanche breakdown operating point.

Using Maxwell's equation in which the particles are the sources for current and charge distribution, the exact field at the position of the particle can be obtained from self-consistent calculation. The kinetic equation becomes

$$
\frac{\partial f_{\alpha}}{\partial t}+\vec{v} \bullet \nabla f_{\infty}+\frac{q}{m}(\overrightarrow{\mathrm{E}}+\vec{v} \times \overrightarrow{\mathrm{B}}) \bullet \nabla_{v} f_{\alpha}=\left(\frac{\partial f_{\alpha}}{\partial t}\right)_{\text {coll }}
$$

where the left hand side contains only averaged quantities and the so-called collision terms on the right hand side contains all microscopic interactions [11]. The collision term for momentum transfer can be evaluated for drifting Maxwell distribution functions, and it is found that

$$
\int_{-\infty}^{+\infty} d v_{\alpha} m v_{\alpha}\left(\frac{\partial f_{\alpha}}{\partial t}\right)_{\text {coll }}=\sum_{\beta} m_{\alpha} n_{\alpha} v_{\alpha \beta}\left(\mu_{\alpha}-\mu_{\beta}\right)
$$

where $\mu_{\alpha}$ and $\mu_{\beta}$ are the drift velocities of species $\alpha$ and $\beta$ [12].

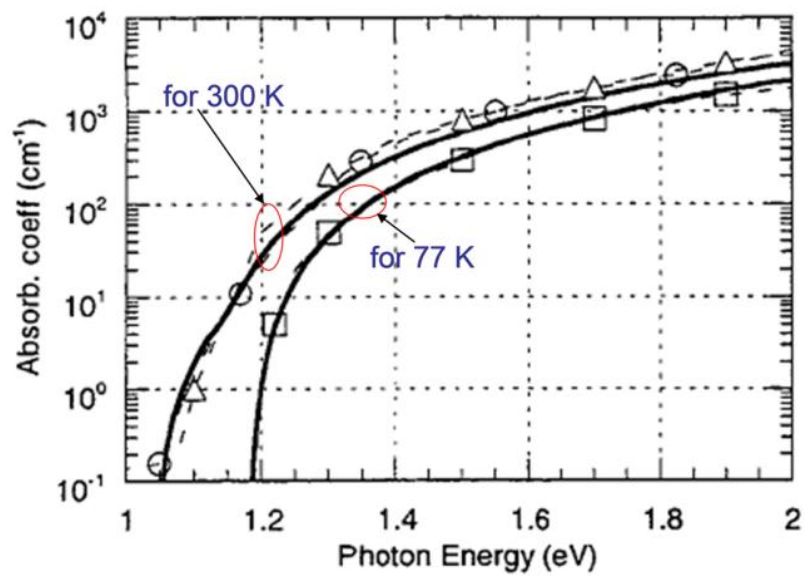

Fig. 8. Optical absorption coefficient of silicon.

Since the photons with energy above $1.08 \sim 1.12 \mathrm{eV}$ (i.e., the silicon energy bandgap lie) are absorbed, the electroluminescence spectra peak shit shown in Fig. 7 should be related to the optical absorption coefficient that has been given by the Macfarlance absorption model [13] , which is also characterized in Fig. 8.

$$
\alpha_{\text {absorption }}=A \cdot\left[\frac{\left(h v-E_{g}-\overline{E_{p n}}\right)}{1-\exp \left(\overline{E_{p n}} / k T\right)}+\frac{\left(h v-E_{g}+\overline{E_{p n}}\right)}{\exp \left(\overline{E_{p n}} / k T\right)-1}\right]
$$

It is further interpreted the spectra obtained under avalanche breakdown conditions, but the multitude of the mechanisms described above shows a great divergence in the interpretation of spectra and proves that the origin of the light emission is not yet well defined and remain in debate [14].

\section{CONCLUSION}

This paper describes the manufacturing and production processes of a micro-system in modern nano-scaled MEMS technology. The potential applications of 600 to $850 \mathrm{~nm} \mathrm{Si}$ LEDs and 600 to $850 \mathrm{~nm}$-based waveguides in CMOS-based optical link toward the micro-photonic systems and MOEMS has been demonstrated. The physical mechanisms responsible for the photon emission identified at some extent to further clarify that the self electro-optic effect of the silicon light source could be further improved for the realization of flip-chip bonded monolithic CMOS optoelectronic smart pixels, in which Fig. 9 presents three different types of structure [15].

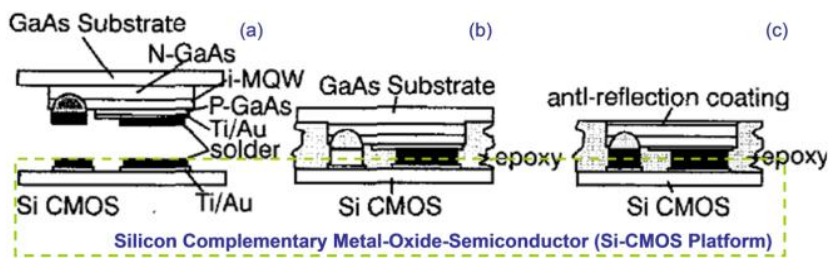

Fig. 9. The flip-chip bonding fabrication procedure.

Finally, the study of multi-terminal low-voltage CMOS $\mathrm{Si}$-LEDs with emission efficiency enhancement is suggested to be analyzed in more detail in near future [16].

\section{ACKNOWLEDGEMENT}

The authors would like to thank the Sichuan Institute of Solid-State Circuits, Chongqing, China, for the assistance in wafer processing, the Chongqing University for help in some of the experiments reported in this paper, and the University of California at Irvine for participating in the electrical characterization and emission microscopy measurements. Prof. Kaikai Xu specially thank Dr. Kingsley A. Ogudo from the University of South Africa, Pretoria, South Africa, and Dr. Carlos Viana from the ESYCOM, ESIEE-Paris, France, and Beiju Huang from the Institute of Semiconductors, Chinese Academy of Sciences, China, for their many contributions to advancing the field of silicon photonics whether their work has been specially cited here or not.

\section{REFERENCES}

[1] L. Snyman, "Development of a 0.75 micron wavelength CMOS optical communication system," Proc. SPIE, vol. 7943, no. 79430K, 2011.

[2] A. Chatterjee, "High-speed light modulation in avalanche breakdown mode for Si diodes," IEEE Electron. Dev. Lett., vol. 25, no. 9, pp. 628-630, 2004.

[3] A. Chynoweth and G. Pearson, "Effect of dislocations on breakdown in silicon p-n junctions," J. Appl. Phys., vol. 29, no. 7, pp. 1103-1110, 1958. 
[4] L. Snyman, "Planar light-emitting electro-optical interfaces in standard silicon complementary metal oxide semiconductor integrated circuitry," Opt. Eng., vol. 41, no. 12, pp. 3230-3240, 2002.

[5] B. Huang, "CMOS monolithic optoelectronic integrated circuit for on-chip optical interconnection," Opt. Commun., vol. 284, no. 16-17, pp. 3924-3927, 2011.

[6] K. Xu, "Electro-optical modulation processes in Si-PMOSFET LEDs operating in the avalanche light emission mode," IEEE Trans. Electron. Dev., vol. 61, no. 6, pp. 2085-2092, 2014.

[7] L. Snyman, H. Aharoni, and M. du Plessis, "Characterization of breakdown phenomena in light emitting silicon $n+p$ diode," J. Appl. Phys., vol. 84, no. 5, pp. 2953-2959, 1998.

[8] L. Snyman, "An efficient low voltage, high frequency silicon CMOS light emitting device and electro-optical interface," IEEE Electron. Dev. Lett., vol. 20, no. 12, pp. 614-617, 1999.

[9] K. Ogudo, "Optical propagation and refraction in silicon complementary metal-oxide semiconductor structures at $750 \mathrm{~nm}$ : Toward on-chip optical links and microphotonic systems," $J$. Micro/Nanolithograph., Microfabrication, Microsyst., vol. 12, no. 1, p. 13015, 2013.

[10] CMOS MOEMS Sensor Device, PCT International Patent Application, PCT/ZA2010/00033, U.S. Patent Application: 20120154812.

[11] K. Xu and G. Li, "Light-emitting device with monolithic integration on bulk silicon in standard complementary metal oxide semiconductor technology," J. Nanophoton., vol. 7, no. 1, p. 73082, 2013.

[12] K. Xu and G. Li, "Hot-carrier induced photon-emission in silicon metal-oxide-semiconductor field-effect-transistor," J. Phys: Conf. Ser. vol. 7, iss. 13, p. 73082, 2013.

[13] J. Polleux and C. Rumelhard, "Optical absorption coefficient determination and physical modeling of strained $\mathrm{SiGe} / \mathrm{Si}$ photodetectors," in Proc. High Perform. Elect. Dev. Micr. and Optoelect. Appl., 8th IEEE Int. Symp., 2000, pp. 167-172.

[14] H. Ghazi, "Analysis of silicon light emission under breakdown condition using an indirect intraband model," Opt. Commun., vol. 281, iss. 12 , pp. 3320-3323, 2008.

[15] H. Chen, "Flip-chip bonded hybrid CMOS/SEED optoelectronic smart pixels," IEE Proc.-Optoelectron., vol. 147, no. 1, pp. 2-6, 2000

[16] M. du Plessis, H. Aharoni, and L. Snyman, "Two- and multi-terminal CMOS/BiCMOS Si LED's," Opt. Mater., vol. 27, no. 5, pp. 1059-1063, 2005.

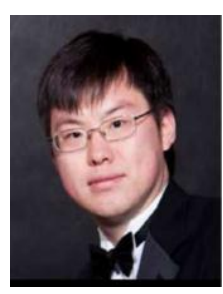

Kaikai Xu was born in Chongqing, China, in 1984 He received the B.S. degree from the University of Electronic Science and Technology of China (UESTC), Chengdu, China, in May 2006, and the Ph.D. degree from the University of California at Irvine (UCI), USA, in June 2014. Since September 2014, he is an Associate Professor with the University of Electronic Science and Technology of China, Chengdu, China. Since December 2014, he is a Distinguished Professor (1000-Talents Program) of Sichuan Province, China. As an international conference Technical/Organizing committee member, he serves for: 20th OptoElectronics and Communications Conference (OECC 2015), 8th International Photonics and OptoElectronics Meetings (POEM 2015), 15th International Conference on Numerical Simulation of Optoelectronic Devices (NUSOD 2015), Conference on Advances in Wireless and Optical Communications (RTUWO 2015), IEEE International Workshop of Electronics, Control, Measurement, Signal and their application to Mechatronics (ECMSM 2015), 17th International Conference on Transparent Optical Networks (ICTON 2015), International Symposium on Photonics and Optoelectronics (SOPO 2015), 4th International Conference on Photonics, Optics and Laser Technology (PHOTOPTICS 2016), 5th International Conference on Sensor Networks (SENSORNETS 2016), 24th Wireless and Optical Communication Conference (WOCC 2015), International Conference on Automation, Cognitive Science, Optics, Micro Electro-Mechanical System, and Information Technology (ICACOMIT 2015), IEEE AFRICON 2015, International Conference on Opto-electronic and Applied Optics (IEM OPTRONIX-2015), and 21st Asia and South Pacific Design Automation Conference (ASP-DAC 2016). As an international journal editor, he serves for: International Journal of Materials, Mechanics and Manufacturing (EI), Journal of Optoelectronics and Advanced Materials (SCI), Optical and Quantum Electronics (SCI), International Journal of Innovative Research in
Science, Engineering and Technology (Scopus), and Optics and Photonics Journal. He is also the General Co-chair for the conference: IEEE Advanced Information Technology, Electronic and Automation Control Conference (IAEAC 2015) and is the Editor-in-Chief for the journal: Advances in Optoelectronic Materials. Most recently, he was awarded an IEEE Photonics Society International Year of Light (IYL 2015) Grant.

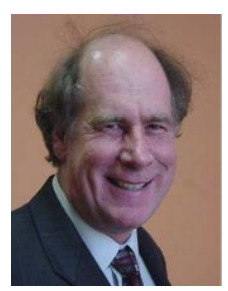

Lukas W. Snyman received a PhD degrees, in semiconductor physics from the University of Port Elizabeth, South Africa in 1987 From 1990 to 2010. he was a an associate Professor at the University of Pretoria and subsequently professor at the Tshwane Technical University of Technology (TUT). Since 2014, he is at the School of Science Engineering and Technology (UNISA -CSET) at University of South Africa in Florida, Johannesburg. He has to date published about 80 scientific articles, mostly in international conference proceedings and scientific journals. $\mathrm{He}$ is author and co-author of two scholarly chapters in Intech books. The latest was in “ Integrating Micro-Photonic Systems and MOEMS into Standard Silicon CMOS Integrated Circuitry". In Optoelectronics and Applications in 2014. $\mathrm{He}$ is the main inventor of four granted U.S.A, co-inventor of two granted USA patents, main inventor of two European Patents, two Korean patents, one Chinese patent, and eleven granted SA patents, mainly on the topics of Si Av LEDs, optoelectronics and photonics. Professor Snyman received an Academic Excellence Award of Innovator of the Year at the TUT in 2010. Most recently, he was a finalist for a prestigious Special Photonics Award: International Year of Light for 2015, as awarded by the National Science and Technology and Billiton (NSTF-Billiton) forum in South Africa. He is a current member of the Institute for Electrical and Electronic Engineers in the USA, a member of the Society for Photonic and Instrumentation Engineers in the USA.

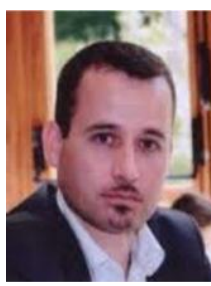

Jean-Luc Polleux received the degree/Diplôme d'ingénieur in microelectronic from ENSEIRB, Bordeaux, France, and the DEA degree in electronic and telecommunications from the University of Bordeaux, France, both in 1997. He received the Ph.D. degree in the opto-microwave field from CNAM, Paris, in 2001. He is currently an associate professor at ESIEE-Paris.

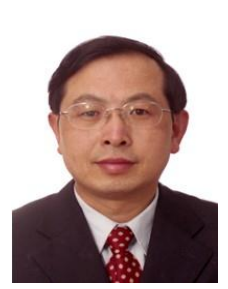

Hongda Chen received his $\mathrm{Ph}$. D. degree from the Tianjin University in 1996 . He is a professor in the Institute of Semiconductors, Chinese Academy of Sciences. He also serves as the deputy director of the same institute. Prof. Chen is a member of IEEE and Optical Society of America, a senior member of the Chinese Institute of Electronics, a member of Semiconductor Committee of Beijing Electronic Academy.

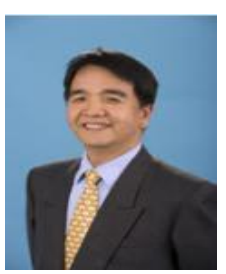

Guannpyng Li obtained his Ph.D. degree from the University of California, Los Angeles, in 1984. He is a professor in the University of California, Irvine (UCI), and the director of the Integrated Nanosystems Research Facility at UCI and the Irvine Division director of the California Institute for Telecommunications and Information Technology. Most recently, he is on the International advisory committee for the International Workshop on Thin-films for Electronics, Electro-Optics, Energy, and Sensors, held by SPIE in 2015 . 